\title{
A novel fully convolutional network for visual saliency prediction
}

\author{
Bashir Muftah Ghariba ${ }^{\text {Corresp., 1, } 2}$, Mohamed S Shehata ${ }^{3}$, Peter McGuire ${ }^{4}$ \\ ${ }^{1}$ Faculty of Engineering \& Applied Science, Memorial University of Newfoundland, St.john's, NL, Canada \\ 2 Department of Electrical and Computer Engineering, Faculty of Engineering, Elmergib University, Khoms, Libya \\ 3 Department of Computer Science, Mathematics, Physics and Statistics, University of British Columbia, Kelowna, BC, Canada \\ 4 C-CORE, St.john's, NL, Canada \\ Corresponding Author: Bashir Muftah Ghariba \\ Email address: bmg063@mun.ca
}

Human Visual System (HVS) has the ability to pay visual attention, which is one of the many functions of the HVS. Despite the many advancements being made in visual saliency prediction, there continues to be room for improvement. Deep learning has recently been used to deal with this task. This paper proposes a novel deep learning model based on a Fully Convolutional Network (FCN) architecture. The proposed model is trained in an endto-end style and designed to predict visual saliency. The entire proposed model is fully training style from scratch to extract distinguishing features. The proposed model is evaluated using several benchmark datasets, such as MIT300, MIT1003, TORONTO, and DUT-OMRON. The quantitative and qualitative experiment analyses demonstrate that the proposed model achieves superior performance for predicting visual saliency. 
1 Article submitted to PeerJ Computer Science

\section{A Novel Fully Convolutional Network for Visual 3 Saliency Prediction}

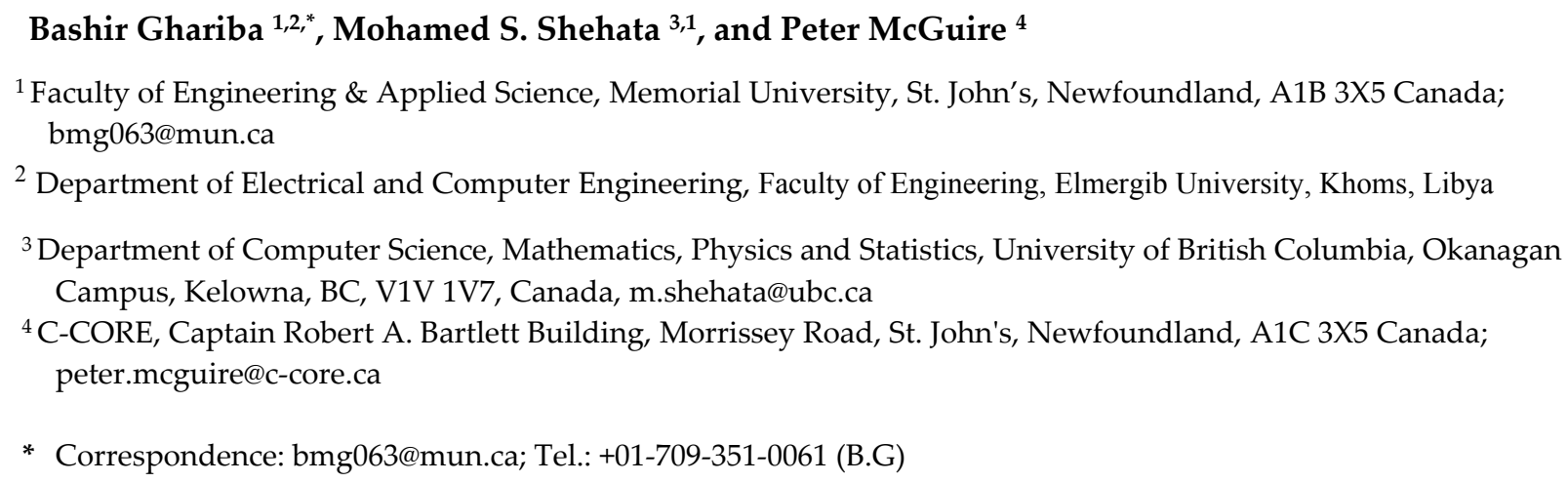

\section{Abstract}

Human Visual System (HVS) has the ability to pay visual attention, which is one of the many functions of the HVS. Despite the many advancements being made in visual saliency prediction, there continues to be room for improvement. Deep learning has recently been used to deal with this task. This paper proposes a novel deep learning model based on a Fully Convolutional Network (FCN) architecture. The proposed model is trained in an end-to-end style and designed to predict visual saliency. The entire proposed model is fully training style from scratch to extract distinguishing features. The proposed model is evaluated using several benchmark datasets, such as MIT300, MIT1003, TORONTO, and DUT-OMRON. The quantitative and qualitative experiment analyses demonstrate that the proposed model achieves superior performance for predicting visual saliency.

Keywords: Deep learning; Convolutional Neural Network; FCN; Semantic segmentation; Encoder-decoder architecture; Human eye fixation; HVA.

\section{Introduction}

A Human Visual System (HVS) processes a part of the visual scene instead of the whole scene. This phenomenon is called Human Visual Attention (HVA), also referred to as visual saliency prediction, which is an important research area in the field of computer vision. HVA is also known as human eye fixation prediction, visual saliency prediction, or saliency map detection. Visual saliency prediction is also beneficial for other applications in the computer vision field, including 
salient object detection (Liu \& Han 2016), image retrieval (Huang et al. 2011), multiresolution imaging (Lu \& Li 2013), and scene classification (Cheng et al. 2015; Lu et al. 2014; Yao et al. 2016).

Many models have been developed to predict visual saliency, the most popular being the saliency map. Saliency maps describe the probability that each image pixel will attract human attention. In other words, saliency maps are images that display the unique qualities of each pixel in a given image (Gao \& Vasconcelos 2005). To produce a saliency map, the salient points in the image are collected and convolved with a Gaussian filter (Gao \& Vasconcelos 2005). The probability that each pixel in the image will attract human attention is represented by a heat map or gray-scale image. Notably, saliency maps smooth the image, making it more meaningful and easier to analyze. This is useful for condition image captioning architecture because it indicates what is salient and what is not (Mackenzie \& Harris 2017).

To evaluate the saliency map, human eye fixation data in free viewing is used because there is a direct link between human eye movement and visual attention (Mackenzie \& Harris 2017). Generally, HVA runs on two approaches. The first is a bottom-up approach which utilizes lowlevel features, including intensity, color, edge orientation, and texture (Gao et al. 2008; Le Meur et al. 2006). Such an approach attempts to decide regions that show obvious characteristics of their surroundings. The second is a top-down approach, which is task-driven and requires an explicit understanding of the context of the visual scene. Moreover, it depends on the features of the object of interest (Gao et al. 2009; Kanan et al. 2009).

The deep Convolutional Neural Network (CNN) is the most widely utilized deep learning method for image processing applications (Mahdianpari et al. 2018). Specifically, CNN is capable of extracting discriminant visual features (e.g., 2-D spatial features) by applying a hierarchy of convolutional filters using multiple nonlinear transformations. Studies have also used Convolutional Neural Networks (CNNs) for studying saliency map detection to confirm the importance of end-to-end task learning and automatic feature extraction (Fang et al. 2016; Jetley et al. 2016; Kruthiventi et al. 2016; Pan et al. 2016; Vig et al. 2014). The deep CNN model achieves an even higher classification accuracy. For example, deep learning techniques have achieved superior results in multiple tasks, such as driverless car, scene classification, object (e.g., vehicle) detection, image classification, and semantic segmentation. However, deep learning architecture requires sufficient training data for superior performance on several sets of visual tasks, such as local image detection (Girshick et al. 2014), global image classification(Krizhevsky et al. 2012), and semantic segmentation (Long et al. 2015).

Although several deep learning models have been proposed to solve the problem of saliency prediction, and those models provide good performance. However, those models essentially proposed for object recognition and then fine-tuned for saliency prediction. Consequently, the pixel-based classification of visual attention task remains challenging. This highlights the 
necessity of designing a novel FCN model specifically for the task of saliency prediction. In addition, our proposed model is designed for training from scratch. Therefore, we added some modules (e.g., three inception modules and residual modules) to improve the model performance.

The inception module is useful since benefits from filters with different sizes in one layer, which contribute to multi-scale inference and enhance contextual information. This highlights the necessity of combining feature maps at different resolution to extract useful information. In addition, residual module recovers more accurate information and simplifies optimization, while avoiding the vanishing gradient problem. Moreover, residual module decreases the number of parameters by drop of several layers in deep learning model. Therefore, prevent the overfitting of the proposed model.

In this study, we utilized an encoder-decoder structure based on the Fully Convolutional Network (FCN) architecture to address the problem of bottom-up visual attention in visual saliency predication. FCN has the same architecture as the CNN network, but unlike CNN it does not contain any fully connected layers. FCNs are also powerful visual models that generate high-level features from low-level features to produce hierarchies. Moreover, FCN utilizes multi-layer information and addresses pixel-based classification tasks using an end-to-end style (Long et al. 2015). In addition, the proposed model also includes both inception and residual modules to improve multi-scale inference and the recovery of more accurate information, respectively.

This study proposes a new model based on an encoder-decoder structure (i.e., FCN) to improve the performance of visual saliency prediction. The specific contributions of this work are as follows:

(1) A new model of FCN architecture for visual saliency prediction that uses two types of modules is proposed. The first module contains three stages of inception modules, improves the multi-scale inference, and performs contextual information. The second module contains one stage from the residual module and also recovers more accurate information and simplifies optimization, while avoiding the vanishing gradient problem.

(2) Four well-known datasets, including TORONTO, MIT300, MIT1003, and DUT-OMRON, were used to evaluate the proposed model. The experiments demonstrate that the proposed model achieves results comparable or superior to those of other state-of-the-art models.

The remainder of this paper is organized as follows. First, the proposed model is described in more detail in Section 2 and the materials and methods used to produce and evaluate the proposed model are discussed in Section 3. Section 4 presents the quantitative and qualitative experimental results obtained from the four datasets. Finally, the results are summarized, and possible future uses and applications of the proposed model are explored in Section 5.

\section{Related Work}


108

109

110

111

112

113

114

115

116

117

118

119

120

121

122

123

124

125

126

127

128

129

130

131

132

133

134

135

136

137

138

139

140

141

142

143

Visual saliency prediction has received attention from computer vision researchers for many years. The earliest computational model was introduced by Koch and Ullman (Krizhevsky et al. 2012), which inspired the work of Itti et al. (Itti et al. 1998). This model combines low level features at multiple scales to generate saliency maps. Subsequently, many models have been proposed to address visual saliency detection (Fu et al. 2015; Gong et al. 2015; Guo et al. 2017; Li et al. 2014; Liu et al. 2016; Liu et al. 2014a; Liu et al. 2014b; Wang \& Shen 2017; Wang et al. 2019a; Wang et al. 2016; Wang et al. 2017b). Most of this work has been focused on how to detect visual saliency in an image/video using different methods (Borji \& Itti 2012; Wang et al. 2017a; Wang et al. 2019b).

Most conventional attention models are based on a bottom-up strategy. These contain three important steps to detect visual saliency: feature extraction, saliency extraction, and saliency combination. Salient regions in the visual scene are first extracted from their surroundings through hand-crafted low-level features (e.g., intensity, color, edge orientation, and texture), and centersurround contrast is widely used for generating saliency. The saliency may also be produced by the relative difference between the region and its local surroundings (Itti et al. 1998), (Harel et al. 2007), (Bruce \& Tsotsos 2006). The last step for saliency detection combines several features to generate the saliency map.

Recently, many visual saliency models have been introduced for object recognition. Deeplearning models achieved better performance compared to non-deep learning models. The Deep Neural Networks (DNN) (Vig et al. 2014), was trained from scratch to predict saliency. Subsequent models were based on pre-trained models, for example, the DeepGaze I model (Kümmerer et al. 2014), which was the first to be trained on a pre-trained model (AlexNet (Krizhevsky et al. 2012) trained on ImageNet (Deng et al. 2009)), and outperformed the training stage from scratch. DeepGaze II (Kümmerer et al. 2016) has also has been proposed based on a pre-trained model (VGG-19 (Simonyan \& Zisserman 2014)), where attention information was extracted from the VGGNet without fine-tuning the attention task. Next, the DeepFix model (Kruthiventi et al. 2017) was proposed by Kruthiventi et al. based on a pre-trained model VGG16. Furthermore, in (Mahadevan \& Vasconcelos 2009) object detection and saliency detection were carried out using a deep convolutional neural network (CNN). Finally, the SALICON net model (Huang et al. 2015) was proposed to capture multi-scale saliency using combined fine and coarse features from two-stream CNNs that were trained with multi-scale inputs.

Since the superior success of transfer learning models for visual saliency prediction has been established, several new models have been proposed that have improved saliency prediction performance. For instance, the SALICON model fine-tunes a mixture of deep features (Huang et al. 2015) using AlexNet (Krizhevsky et al. 2012), VGG-16 network (Simonyan \& Zisserman 2014), and GoogleNet (Szegedy et al. 2015) for visual saliency prediction. PDP (Jetley et al. 2016) 
144

145

146

147

148

149

150

151

152

153

154

155

156

157

158

159

160

161

162

163

164

165

166

167

168

169

170

171

172

173

174

175

176

177

178

179

180

and DeepFix (Kruthiventi et al. 2017) were used on the VGG-19 network for the same task using MIT300 and the SALICON dataset, and FUCOS (Bruce et al. 2016) fine-tunes features that were trained on the PASCAL dataset. Overall, DeepFix and SALICON models demonstrated significantly improved performance compared to DeepGaze I in the MIT benchmark.

\section{Material and Methods}

\subsection{Proposed model}

The proposed model follows an FCN structure (i.e., a pixel-based approach) and the generic encoder-decoder form. The important difference between CNN and FCN networks is that the latter has learning filters throughout its structure. Even the decision-making layers at the end of the network are filters. FCNs also do not have any fully connected layers that are usually available at the end of the network.

Figure 1 explains the architecture of the proposed model for visual saliency prediction and the configuration of the proposed model is explained in Table 1. The encoder stage contains three blocks of convolution layers, each of which is followed by batch normalization, rectified linear unit (ReLU), and max pooling. The encoder stage is the same as that of a conventional CNN and generates feature maps by down-sample pooling. The decoder stage also transposes convolutional layers but does so in the opposite direction. Therefore, the decoder stage produces label maps (upsampling) with the same input image size. The transposed convolution layers contain un-pooling and convolution operators. Unlike the max-pooling operation, the un-pooling operation increases the size of feature maps through the decoding stage. In addition, the image input size of the proposed model is $224 \times 224$ pixels. Figure 1 illustrates the proposed model architecture to predict visual saliency.

Three inception modules are also used in the proposed model. Inception modules are useful because they benefit from different sized filters in one layer, which contributes to the multi-scale inference and enhances contextual information (Long et al. 2015). In addition, a residual module is also added to the proposed model because it effectively avoids the vanishing gradient problem by introducing an identity shortcut connection (Lin et al. 2014). Moreover, activations from a previous layer are reused by the residual module for the adjacent layer to learn its weights. Figure 2 shows the architecture of the inception and residual modules, respectively. Figure 2 (a) explains the layers of the inception module which contains three branches. The first two contain a sequence of two convolution filters, where the patch sizes of the layers are $1 \times 1$, the second layer is $3 \times 3$, and the last layer is $5 \times 5$, respectively. The third branch contains only one convolutional filter which has a patch size of $1 \times 1$. Each convolutional layer is followed by batch normalization and ReLU. Figure 2 (b) explains the structure of the residual module, which contains two branches. The first branch has a stack of three convolutional filters, sized $1 \times 1,3 \times 3$, and $1 \times 1$, respectively. The second branch has a single $1 \times 1$ convolutional filter. The two branches are combined by element-wise summation. Table 2 explains the number of each filter in the two modules (i.e., inception and 
181 residual). Notably, the convolutional module contains a Convolutional 2D, Batch Normalization,

182 as well as a ReLU layer. The transposed convolutional module also contains the same layers as 183 the convolutional module.

184

185

186

187

188

189

190

191

192

193

194

195

196

197

198

199

200

201

202

203

204

205

206

207

208

209

210

211
Figure 1. Architecture of the proposed model.

Table 1.Configuration of the proposed model.

Note $(*)$ : See Table 2 for the filter size of the inception and residual modules.

Table 2. Configuration of inception and residual modules.

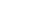

4

Figure 2. Architecture of (a) Inception and (b) residual modules.

\subsection{Semantic Segemnation}

The segmentation task plays an important role in image understanding and is essential for image analysis tasks (Karami et al. 2018). In semantic segmentation, each region or pixel is labeled as a class, such as flower, person, road, sky, ocean, or car. Many applications use semantic segmentation techniques, such as autonomous driving, Bio Medical Image Diagnosis, robotic navigation, localization, and scene understanding. Furthermore, Deep Neural Networks (DNNs) are commonly used as effective techniques for semantic segmentation (Long et al. 2015). Semantic segmentation works with semantics and location; global information determines the "what" while local information determines the "where" of an image. Deep feature hierarchies encode semantics and location in a nonlinear local-to-global pyramid (Long et al. 2015). Our proposed model (i.e., FCN) uses semantic segmentation techniques to assign each pixel in the given image into appropriate classes (i.e., foreground or background) in order to predict visual saliency (i.e., saliency map generation).

\subsection{Datasets}

The proposed model was trained using a standard available dataset (i.e., SALICON) and subsequently tested on four other well-established datasets, including TORONTO, MIT 300, 
212 MIT1003, and DUT-OMRON. All these datasets have different characteristics and so each is

213 described below.

214 3.3.1. SALICON

215 The largest dataset for visual attention applications on the popular Microsoft Common Objects 216 in Context (MS COCO) image database is SALICON (Lin et al. 2014). This dataset contains 21710,000 training, 5,000 validation, and 5,000 testing images with a fixed resolution of 480x640. 218 While this dataset contains the ground truth data for the training and validation datasets, the test 219 dataset ground truth data were unavailable (Jiang et al. 2015).

\section{3.3.2. TORONTO}

221 One of the most widely used datasets for visual attention is the TORONTO dataset. It has 120 222 color images with a resolution of $511 \times 681$ pixels. This dataset contains images that were captured 223 in indoor and outdoor environments and has been free-viewed by 20 human subjects (Bruce \& 224 Tsotsos 2006).

225 3.3.3. MIT300

226 The MIT300 dataset has 300 natural images and the eye-tracking data of 39 users who free 227 viewed these images were used to generate saliency maps. This dataset is challenging since its 228 images are highly variable and natural (Judd et al. 2012). A MIT saliency benchmark website for 229 model evaluation (http://saliency.mit.edu/results_mit300.html) is available to evaluate any 230 saliency model using this dataset.

231

232

233

234

235

236

237

238

239

240

241

242

243

\subsubsection{MIT1003}

MIT1003 includes 1,003 images from the Flicker and LabelMe collections. Saliency maps of these images have also been generated from the eye-tracking data of 15 users. This dataset contains 779 landscape and 228 portrait images that vary in size from $405 \times 405$ to $1024 \times 1024$ pixels, making it the largest available eye fixation dataset (Judd et al. 2009).

\subsubsection{DUT-OMRON}

DUT-OMRON has 5,168 high quality images that were manually selected from over 140,000 images. The largest height or width of this dataset is 400 pixels and each image is represented by five subjects. There is more than one salient object in this type of dataset and the image has a more a complex background (Riche et al. 2013).

\subsection{Evaluation Metrics}

Several methods may be used to evaluate the correspondence between human eye fixation and model prediction (Ghariba et al. 2019). Generally, saliency evaluation metrics are divided into 
244 distribution- and location-based metrics. Previous studies on saliency metrics found it is difficult 245 to perform a reasonable comparison for assessing saliency models using a single metric (Riche et 246 al. 2013). Here, we accomplished our experiment by extensively considering several different 247 metrics, including the Similarity Metric (SIM), Normalized Scanpath Saliency (NSS), and AUC. 248 The last metric is the area under the receiver operating characteristic (ROC) curve (e.g., AUC249 Borji, and AUC-Judd). For clarification, we indicate the map of fixation locations as Q, the 250 predicted saliency map as S, and the continuous saliency map (distribution) as G.

251 3.4.1 Similarity Metric (SIM)

252 The SIM metric produces a histogram that is a measurement of the similarity between two 253 distributions. This metric considers the normalized probability distributions of both the saliency 254 and human eye fixation maps. SIM is also computed as the sum of the minimum values at each 255 pixel, after normalizing the input maps. Equation (1) explains how to calculate the SIM metric.

$$
\mathrm{SIM}=\sum_{\mathrm{i}=1} \min \left(\dot{S}(\mathrm{i}), \mathrm{G}^{(\mathrm{i})}\right),
$$

where

$$
\sum_{\mathrm{i}} \dot{S}^{\prime}(\mathrm{i})=1 \text {, and } \sum_{\mathrm{i}} \dot{G}(\mathrm{i})=1 \text {, }
$$

256

257

258

259

260

261

262

263

264

265 where

and $\mathbf{S}$ and $\mathbf{G}$ are the normalized saliency and the fixation maps, respectively. Importantly, a similarity of one indicates that the distributions are the same whereas a zero indicates that they do not overlap.

\subsubsection{Normalized Scanpath Saliency (NSS)}

NSS was is a simple correspondence measure between saliency maps and ground truth data, computed as the average normalized saliency at fixated locations. NSS is, however, susceptible to false positives and relative differences in saliency across the image (Bylinskii et al. 2018). To calculate NSS given a saliency map S and a binary map of fixation location F,

$$
\mathrm{NSS}=\frac{1}{\mathrm{~N}} \sum_{\mathrm{i}=1}^{\mathrm{N}} \overline{\mathrm{S}}(\mathrm{i}) \times \mathrm{F}(\mathrm{i})
$$

and $\mathrm{N}$ is the total number of human eye positions and $\sigma(S)$ is the standard deviation. 
The AUC-Borji metric, based on Ali Borji's code (Borji et al. 2013), uses a uniform random sample of image pixels as negatives and defines false positives as any fixation (saliency) map values above the threshold of these pixels. The saliency map is a binary classifier that separates positive from negative samples at varying thresholds, the values of which are sampled at a fixed step size. The proportion of the saliency map values above the threshold at the fixation locations is the true positive (TP) rate. Conversely, the proportion of the saliency map values that occur above the threshold sampled from random pixels (as many samples as fixations, sampled uniformly from all image pixels) is the false positive rate (FP).

\subsubsection{AUC-Judd}

The AUC-Judd metric (Judd et al. 2009) is also popular for the evaluation of saliency models. As with AUC-Borji, positive and negative samples are separated at various thresholds by treating the saliency map as a binary classifier. Unlike AUC-Borji, however, the thresholds are sampled from the saliency map's values. The proportion of the saliency map's values above a specific threshold at specific fixation locations is known as the true positive (tp) rate. Alternatively, the proportion of the saliency map's values that occur above the threshold of non-fixated pixels is the false positive (fp) rate.

\section{Experimental Results}

This Section explains all the steps for implementing our work (see Table 3 for more details about experimental steps). Specifically, training, adjusting the parameters, validating, and testing the proposed model on the aforementioned datasets (e.g., TORONTO, MIT300, MIT1003, and DUT-OMRON) are described in details.

\subsection{Model Training}

The most important step for the proposed model is model training. In this work, the proposed model was trained from scratch (i.e., full-training). Training of models from scratch is challenging due to computational and data availability, leading to problems of overfitting. However, there are several techniques, such as normalization, data augmentation, and dropout layers that are useful for mitigating the problems generated from overfitting.

The full-training style has two different categories. In the first category, the CNN architecture is fully designed and trained from scratch. In this case, the number of $\mathrm{CNN}$, pooling layers, the kind of activation function, neurons, learning rate, and the number of iterations should be determined. In the second category, the network architecture and the number of parameters remain unchanged, but the advantages of pre-existing architecture and full-training is applied to given images. 
301

302

303

304

305

306

307

308

309

310

311

312

313

314

315

316

317

318

319

320

321

322

323

324

325

326

327

328

329

330
In this study, the first category was employed. Specifically, the proposed model was trained using the well-known dataset, SALICON (see Section 3.3.1 for more details) and was also validated using a specific validation dataset (i.e., 5000 images). This dataset is the largest available for visual attention (i.e., 10,000 images for training, 5,000 for validation) and was created for saliency applications. At the beginning of the training task, all filter weights were randomly initialized because a pre-trained network was not used in this study. A mini-batch of 16 images was used in each iteration and the learning rate was set as 0.001 . The proposed model parameters were learned using the back-propagating loss function by stochastic gradient descent with a momentum (SGDM) optimizer.

Since the number of images available for the training task was limited (i.e., 10,000 images), we suggested using the date augmentation technique to increase the number of training images by creating modified versions of images in the dataset. This technique was carried out to mitigate overfitting by rotating at $30^{\circ}$ intervals. This technique also improves performance and the proposed model's ability for generalization. Figure 3 illustrates the proposed model's training progress from the mentioned training images (SALICON).

6

7

18


332 5. Discussion

333 5.1. Quantitative Comparison of the Proposed Model with Other Advanced Models

334

335

336

337

338

339

340

341

342

343

344

345

346

347

348

349

350

351

352

353

354

355

356

357

358

359

360

361

362

363

364

To evaluate the efficiency of the proposed model for predicting visual saliency, we compared it to 10 state-of-the-art models, ITT, AIM, Judd Model, GBVS, Mr-CNN, CAS, SalGAN, DeepGaze I, DeepGaze II, and ML-NET. The models were applied to four datasets (i.e., TORONTO, MIT300, MIT1003, and DUT-OMRON), and the quantitative results are presented in Tables 3, 4, 5, and 6, respectively. All these models differ in terms of computational speed (i.e., run time). Table 7 explains the runtime properties of the proposed model as well as the other 10 visual saliency models. From this table, one can see the run time of the proposed model is about $12 \mathrm{~s}$ on our machine which has no GPU and is an Intel CPU i7-3370K.

Notably, the main difference between the proposed model and other state-of-art models is that the proposed model was specifically designed for saliency prediction, whereas the other pretrained models were essentially designed for object recognition and then fine-tuned for the visual saliency prediction task. In addition, the proposed model was trained from scratch, which requires a large number of training images to provide a reasonable performance; however, the largest dataset available for this application contains only 10,000 images (e.g., SALICON), which is considered relatively small to train a model from scratch.

Table 3 shows that, with the TORONTO dataset, the proposed model outperforms other models (deep and classical models) in terms of NSS; however, in terms of SIM, AUC-Judd, and AUC-Borji, the GBVS model provides the best results (note that the bolded values are the best results). From Table 4, one can see that with the MIT300 dataset, the model that provides the best performance is DeepGaz II in terms of the AUC-Judd and AUC-Borji metrics. However, the SalGAN model produces the best results for the SIM metric, while the ML-NET model provides the best value for the NSS metric. In Table 5 (for MIT1003 dataset), one can see that the proposed model surpasses the other models in terms of the SIM and AUC-Judd metrics, while the GBVS model provides the best results for the NSS metric. Finally, Table 6 shows that, with the DUTOMRON dataset, the proposed model achieved the best result in terms of the AUC-Judd metric, while the GBVS model is the best in terms of the AUC-Borji metric.

Table 3. Comparison of the quantitative scores of several models on TORONTO (Bruce \& Tsotsos 2006) dataset.
Note. Humans baseline
3.29
1.00
0.92
0.88

Table 4. Comparison of the quantitative scores of several models on MIT300 (Judd et al. 2012) dataset. 
366 Table 5. Comparison of the quantitative scores of several models on MIT1003(Judd et al. 2009)

367 dataset.

368

369

370

371

372

373

374

375

376

377

378

379

380

381

382

383

384

385

386

387

388

389

390

391

392

Table 6. Comparison of the quantitative scores of several models on DUT-OMRON (Yang et al. 2013) dataset.

Table 7: Runtime of the proposed model and ten visual saliency models

\subsection{Qualitative Comparison of the Proposed Model with Other Advanced Models}

The qualitative results obtained by the proposed model are compared with five state-of-the art models, ITTI, FES, CovSal, GBVS, and SDS-GM (Li \& Mou 2019), on the aforementioned datasets (i.e., TORONTO, MIT300, MIT1003, and DUT-OMRON). Figure 4 shows the visual saliency map results and the proposed model visual saliency prediction, i.e., generating saliency map, within the given images. Based on the evaluation of the proposed model, the proposed model produces saliency maps comparable to other state-of-the-art models.

Figure 4. Saliency maps obtained from the proposed model and five advanced models for a sample image from the TORONTO, MIT300, MIT1003, and DUT-OMRON datasets.

\subsection{Ablation Study}

In this work, we evaluated several different aspects of the proposed model's architecture. Table 8 illustrates the results of the experiments conducted in this work. Based on the architecture of the proposed model, we suggested 13 different scenarios in order to find an optimum architecture. Several conclusions were obtained based on these experiments:

(1) From scenarios S1 to S4, we can see the best global accuracy is achieved with 3 encoder-3 decoder stages (i.e., global accuracy was $85.05 \%$ and loss function was 0.2384 ).

(2) S7 describes the proposed model using 3 convolutional modules \& 3 inception modules. This architecture also produced the best global accuracy (i.e., global accuracy was $93.63 \%$, and loss 
393 function was 0.1051 ) compared to S5 and S6, which contain one and two inception modules, 394 respectively.

395 (3) S13 is the last scenario we selected as the entire model, including 3 convolutional, 3 inception,

396

397

398

399

400

401

402

403

404

405

406

407

408

409

410

411

412

413

414

415

416

417

418

419

420

421

422

423

424

425

426

427 and 1 residual module (i.e., Figure 1). This scenario produced a higher global accuracy (i.e., global accuracy was $97.05 \%$, and loss function was 0.07 ) compare to those of scenarios S11 and S12.

Table 8. Different FCN models applied in this study.

\section{Conclusions}

A new deep $\mathrm{CNN}$ model has been proposed in this paper for predicting visual saliency in the field of view. The main novelty of this model is its use of a new deep learning network with three encoders and three decoders (convolution and deconvolution) for visual saliency prediction, as well as its inclusion of two modules (inception and residual modules). The proposed model was trained from scratch and used the data augmentation technique to produce variations of images. The experiment results illustrate that the proposed model achieves superior performance relative to other state-of-the-art models. Moreover, we discovered that an increase in the number of training images will increase the model prediction accuracy (i.e., improvement in model performance); however, the implementation of the model requires a large amount of memory and so it is difficult to use large numbers of training images. Furthermore, because the model was trained from scratch, we expected the model will require more training data that other models, which are currently unavailable.

A promising direction for future research is to collect a new dataset, generate its ground truth, and design new models with good performance and improved evaluation metrics based on the one proposed herein. Extending the proposed model and applying it to examples of dynamic saliency (i.e., video images), is another plausible and interesting avenue of research. The proposed model may also facilitate other tasks, such as scene classification, salient object detection, and object detection, making it applicable in a number of disciplines. Importantly, future models based on that proposed herein should be able to learn from high-level understanding, so they are able to, for example, detect the most important object of the image (e.g., focusing on the most important person in the room). Saliency models also need to understand high-level semantics in the visual scene (i.e., semantic gap), and cognitive attention studies can help to overcome some of the restrictions identified in the proposed model.

\section{Acknowledgements}

The authors acknowledge the support of the Libyan Ministry of Higher Education and Scientific Research, and Elmergib University, Alkhums. 


\section{References}

431

432

433

434

435

436

437

438

439

440

441

442

443

444

445

446

447

448

449

450

451

452

453

454

455

456

457

458

459

460

461

462

463

464
Borji A, and Itti L. 2012. State-of-the-art in visual attention modeling. IEEE transactions on pattern analysis and machine intelligence 35:185-207.

Borji A, Tavakoli HR, Sihite DN, and Itti L. 2013. Analysis of scores, datasets, and models in visual saliency prediction. Proceedings of the IEEE international conference on computer vision. p 921-928.

Bruce N, and Tsotsos J. 2006. Saliency based on information maximization. Advances in neural information processing systems. $p$ 155-162.

Bruce ND, Catton C, and Janjic S. 2016. A deeper look at saliency: Feature contrast, semantics, and beyond. Proceedings of the IEEE Conference on Computer Vision and Pattern Recognition. p 516-524.

Bylinskii Z, Judd T, Oliva A, Torralba A, and Durand F. 2018. What do different evaluation metrics tell us about saliency models? IEEE transactions on pattern analysis and machine intelligence 41:740757.

Cheng G, Han J, Guo L, Liu Z, Bu S, and Ren J. 2015. Effective and efficient midlevel visual elementsoriented land-use classification using VHR remote sensing images. IEEE Transactions on Geoscience and Remote Sensing 53:4238-4249.

Deng J, Dong W, Socher R, Li L-J, Li K, and Fei-Fei L. 2009. Imagenet: A large-scale hierarchical image database. 2009 IEEE conference on computer vision and pattern recognition: leee. p 248-255.

Fang S, Li J, Tian Y, Huang T, and Chen X. 2016. Learning discriminative subspaces on random contrasts for image saliency analysis. IEEE transactions on neural networks and learning systems 28:1095-1108.

Fu K, Gong C, Gu IY-H, and Yang J. 2015. Normalized cut-based saliency detection by adaptive multi-level region merging. IEEE Transactions on Image Processing 24:5671-5683.

Gao D, Han S, and Vasconcelos N. 2009. Discriminant saliency, the detection of suspicious coincidences, and applications to visual recognition. IEEE transactions on pattern analysis and machine intelligence 31:989-1005.

Gao D, Mahadevan V, and Vasconcelos N. 2008. The discriminant center-surround hypothesis for bottomup saliency. Advances in neural information processing systems. p 497-504.

Gao D, and Vasconcelos N. 2005. Discriminant saliency for visual recognition from cluttered scenes. Advances in neural information processing systems. $p$ 481-488.

Ghariba B, Shehata MS, and McGuire P. 2019. Visual Saliency Prediction Based on Deep Learning. Information 10:257.

Girshick R, Donahue J, Darrell T, and Malik J. 2014. Rich feature hierarchies for accurate object detection and semantic segmentation. Proceedings of the IEEE conference on computer vision and pattern recognition. p 580-587.

Peer] Comput. Sci. reviewing PDF | (CS-2019:11:43453:2:0:NEW 30 May 2020) 
465

466

467

468

469

470

471

472

473

474

475

476

477

478

479

480

481

482

483

484

485

486

487

488

489

490

491

492

493

494

495

496

497

498

499

500

501

Gong C, Tao D, Liu W, Maybank SJ, Fang M, Fu K, and Yang J. 2015. Saliency propagation from simple to difficult. Proceedings of the IEEE conference on computer vision and pattern recognition. $p$ 25312539.

Guo F, Wang W, Shen J, Shao L, Yang J, Tao D, and Tang YY. 2017. Video saliency detection using object proposals. IEEE Transactions on Cybernetics 48:3159-3170.

Harel J, Koch C, and Perona P. 2007. Graph-based visual saliency. Advances in neural information processing systems. $p$ 545-552.

Huang J, Yang X, Fang X, Lin W, and Zhang R. 2011. Integrating visual saliency and consistency for reranking image search results. IEEE Transactions on Multimedia 13:653-661.

Huang X, Shen C, Boix X, and Zhao Q. 2015. Salicon: Reducing the semantic gap in saliency prediction by adapting deep neural networks. Proceedings of the IEEE International Conference on Computer Vision. p 262-270.

Itti L, Koch C, and Niebur E. 1998. A model of saliency-based visual attention for rapid scene analysis. IEEE transactions on pattern analysis and machine intelligence 20:1254-1259.

Jetley S, Murray N, and Vig E. 2016. End-to-end saliency mapping via probability distribution prediction. Proceedings of the IEEE Conference on Computer Vision and Pattern Recognition. p 5753-5761.

Jiang M, Huang S, Duan J, and Zhao Q. 2015. Salicon: Saliency in context. Proceedings of the IEEE conference on computer vision and pattern recognition. $p$ 1072-1080.

Judd T, Durand F, and Torralba A. 2012. A benchmark of computational models of saliency to predict human fixations.

Judd T, Ehinger K, Durand F, and Torralba A. 2009. Learning to predict where humans look. 2009 IEEE 12th international conference on computer vision: IEEE. p 2106-2113.

Kanan C, Tong MH, Zhang L, and Cottrell GW. 2009. SUN: Top-down saliency using natural statistics. Visual Cognition 17:979-1003.

Karami E, Shehata MS, and Smith A. 2018. Adaptive polar active contour for segmentation and tracking in ultrasound videos. IEEE Transactions on Circuits and Systems for Video Technology 29:1209-1222.

Krizhevsky A, Sutskever I, and Hinton GE. 2012. Imagenet classification with deep convolutional neural networks. Advances in neural information processing systems. p 1097-1105.

Kruthiventi SS, Ayush K, and Babu RV. 2017. Deepfix: A fully convolutional neural network for predicting human eye fixations. IEEE Transactions on Image Processing 26:4446-4456.

Kruthiventi SS, Gudisa V, Dholakiya JH, and Venkatesh Babu R. 2016. Saliency unified: A deep architecture for simultaneous eye fixation prediction and salient object segmentation. Proceedings of the IEEE Conference on Computer Vision and Pattern Recognition. p 5781-5790.

Kümmerer M, Theis L, and Bethge M. 2014. Deep gaze i: Boosting saliency prediction with feature maps trained on imagenet. arXiv preprint arXiv:14111045.

Kümmerer M, Wallis TS, and Bethge M. 2016. DeepGaze II: Reading fixations from deep features trained on object recognition. arXiv preprint arXiv:161001563.

Peer] Comput. Sci. reviewing PDF | (CS-2019:11:43453:2:0:NEW 30 May 2020) 
502

503

504

505

506

507

508

509

510

511

512

513

514

515

516

517

518

519

520

521

522

523

524

525

526

527

528

529

530

531

532

533

534

535

536

537

538

Le Meur O, Le Callet P, Barba D, and Thoreau D. 2006. A coherent computational approach to model bottom-up visual attention. IEEE transactions on pattern analysis and machine intelligence 28:802-817.

Li Y, Hou X, Koch C, Rehg JM, and Yuille AL. 2014. The secrets of salient object segmentation. Proceedings of the IEEE Conference on Computer Vision and Pattern Recognition. p 280-287.

Li Y, and Mou X. 2019. Saliency detection based on structural dissimilarity induced by image quality assessment model. Journal of Electronic Imaging 28:023025.

Lin T-Y, Maire M, Belongie S, Hays J, Perona P, Ramanan D, Dollár P, and Zitnick CL. 2014. Microsoft coco: Common objects in context. European conference on computer vision: Springer. p 740-755.

Liu N, and Han J. 2016. Dhsnet: Deep hierarchical saliency network for salient object detection. Proceedings of the IEEE Conference on Computer Vision and Pattern Recognition. p 678-686.

Liu N, Han J, Liu T, and Li X. 2016. Learning to predict eye fixations via multiresolution convolutional neural networks. IEEE transactions on neural networks and learning systems 29:392-404.

Liu Z, Zhang X, Luo S, and Le Meur O. 2014a. Superpixel-based spatiotemporal saliency detection. IEEE Transactions on Circuits and Systems for Video Technology 24:1522-1540.

Liu Z, Zou W, and Le Meur O. 2014b. Saliency tree: A novel saliency detection framework. IEEE Transactions on Image Processing 23:1937-1952.

Long J, Shelhamer E, and Darrell T. 2015. Fully convolutional networks for semantic segmentation. Proceedings of the IEEE conference on computer vision and pattern recognition. $p$ 3431-3440.

Lu X, and Li X. 2013. Multiresolution imaging. IEEE Transactions on Cybernetics 44:149-160.

Lu X, Li X, and Mou L. 2014. Semi-supervised multitask learning for scene recognition. IEEE Transactions on Cybernetics 45:1967-1976.

Mackenzie AK, and Harris JM. 2017. A link between attentional function, effective eye movements, and driving ability. Journal of experimental psychology: human perception and performance 43:381.

Mahadevan V, and Vasconcelos N. 2009. Saliency-based discriminant tracking. 2009 IEEE conference on computer vision and pattern recognition: IEEE. p 1007-1013.

Mahdianpari M, Salehi B, Rezaee M, Mohammadimanesh F, and Zhang Y. 2018. Very deep convolutional neural networks for complex land cover mapping using multispectral remote sensing imagery. Remote Sensing 10:1119.

Pan J, Sayrol E, Giro-i-Nieto X, McGuinness K, and O'Connor NE. 2016. Shallow and deep convolutional networks for saliency prediction. Proceedings of the IEEE Conference on Computer Vision and Pattern Recognition. p 598-606.

Riche N, Duvinage M, Mancas M, Gosselin B, and Dutoit T. 2013. Saliency and human fixations: State-ofthe-art and study of comparison metrics. Proceedings of the IEEE international conference on computer vision. $p$ 1153-1160.

Simonyan K, and Zisserman A. 2014. Very deep convolutional networks for large-scale image recognition. arXiv preprint arXiv:14091556. 
539

540

541

542

543

544

545

546

547

548

549

550

551

552

553

554

555

556

557

558

559

560

561

Szegedy C, Liu W, Jia Y, Sermanet P, Reed S, Anguelov D, Erhan D, Vanhoucke V, and Rabinovich A. 2015. Going deeper with convolutions. Proceedings of the IEEE conference on computer vision and pattern recognition. p 1-9.

Vig E, Dorr M, and Cox D. 2014. Large-scale optimization of hierarchical features for saliency prediction in natural images. Proceedings of the IEEE Conference on Computer Vision and Pattern Recognition. p 2798-2805.

Wang W, and Shen J. 2017. Deep visual attention prediction. IEEE Transactions on Image Processing 27:2368-2378.

Wang W, Shen J, Dong X, Borji A, and Yang R. 2019a. Inferring salient objects from human fixations. IEEE transactions on pattern analysis and machine intelligence.

Wang W, Shen J, and Shao L. 2017a. Video salient object detection via fully convolutional networks. IEEE Transactions on Image Processing 27:38-49.

Wang W, Shen J, Shao L, and Porikli F. 2016. Correspondence driven saliency transfer. IEEE Transactions on Image Processing 25:5025-5034.

Wang W, Shen J, Xie J, Cheng M-M, Ling H, and Borji A. 2019b. Revisiting video saliency prediction in the deep learning era. IEEE transactions on pattern analysis and machine intelligence.

Wang W, Shen J, Yang R, and Porikli F. 2017b. Saliency-aware video object segmentation. IEEE transactions on pattern analysis and machine intelligence 40:20-33.

Yang C, Zhang L, Lu H, Ruan X, and Yang M-H. 2013. Saliency detection via graph-based manifold ranking. Proceedings of the IEEE conference on computer vision and pattern recognition. $p$ 3166-3173.

Yao X, Han J, Cheng G, Qian X, and Guo L. 2016. Semantic annotation of high-resolution satellite images via weakly supervised learning. IEEE Transactions on Geoscience and Remote Sensing 54:36603671.

562 


\section{Figure 1}

\section{Architecture of the proposed model}

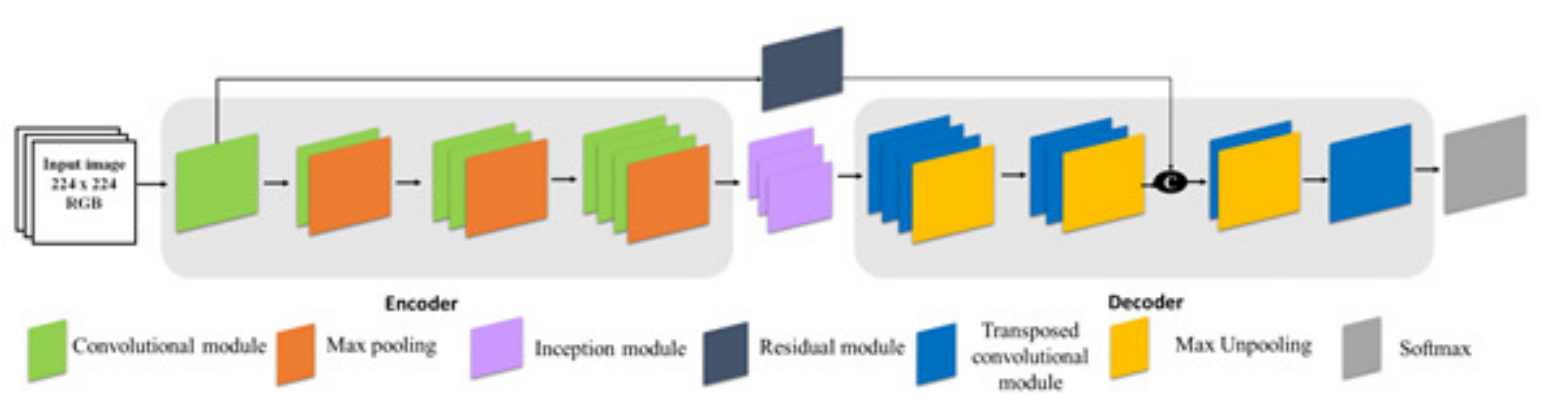

(- Conatenatico 


\section{Figure 2}

Architecture of (a) Inception and (b) residual modules

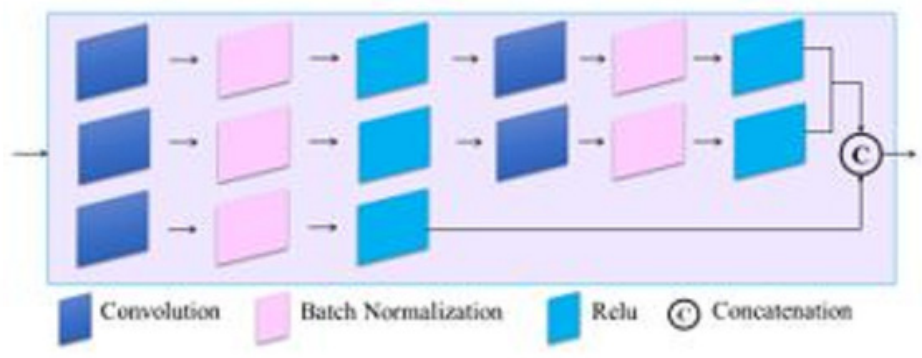

(A)

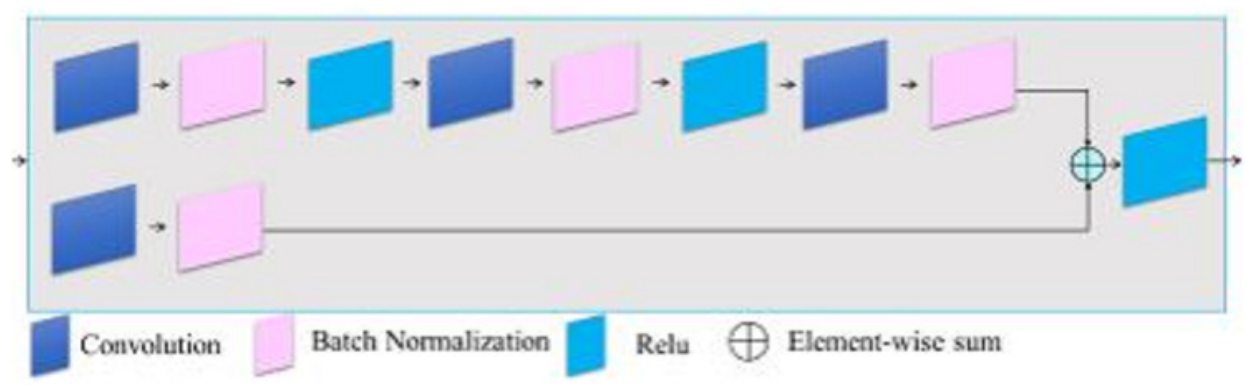

(B) 
Figure 3

Value of validation accuracy (a) and loss as a function of epochs (b) 


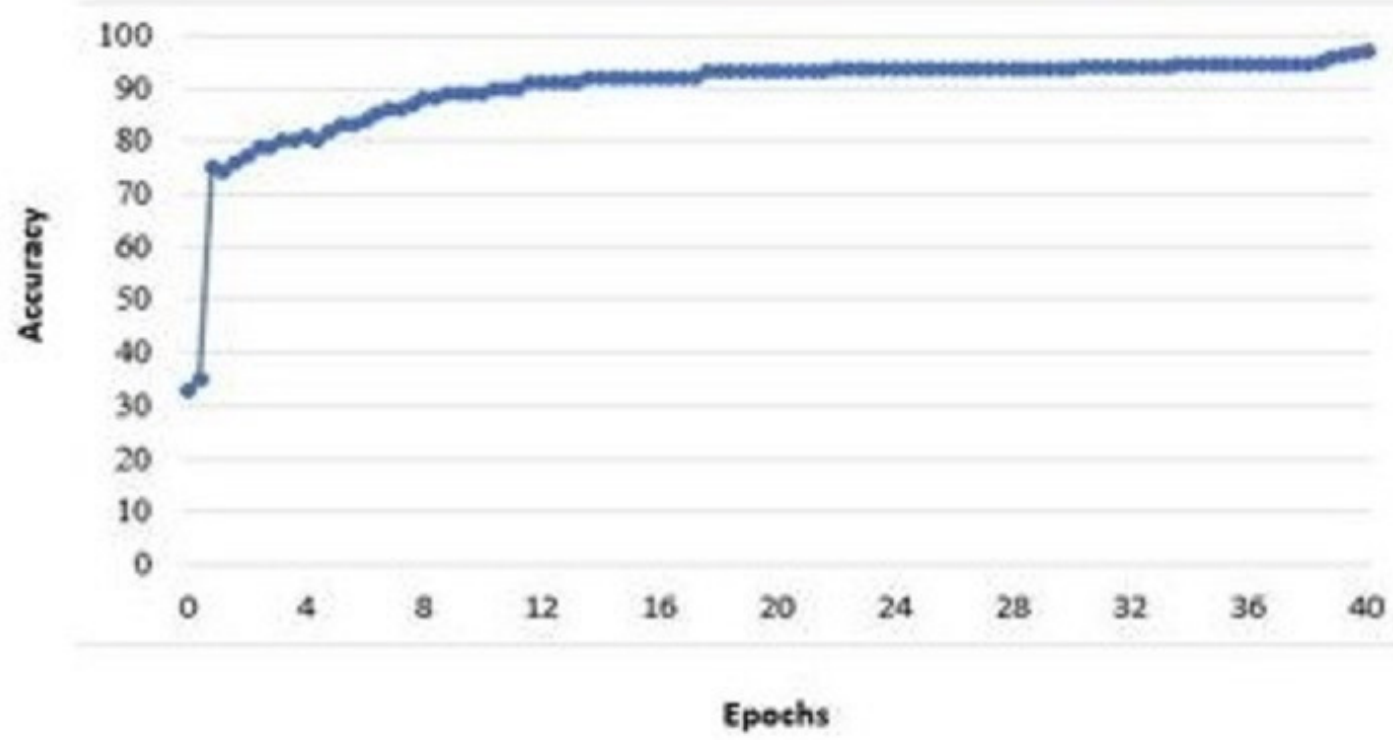

(A)

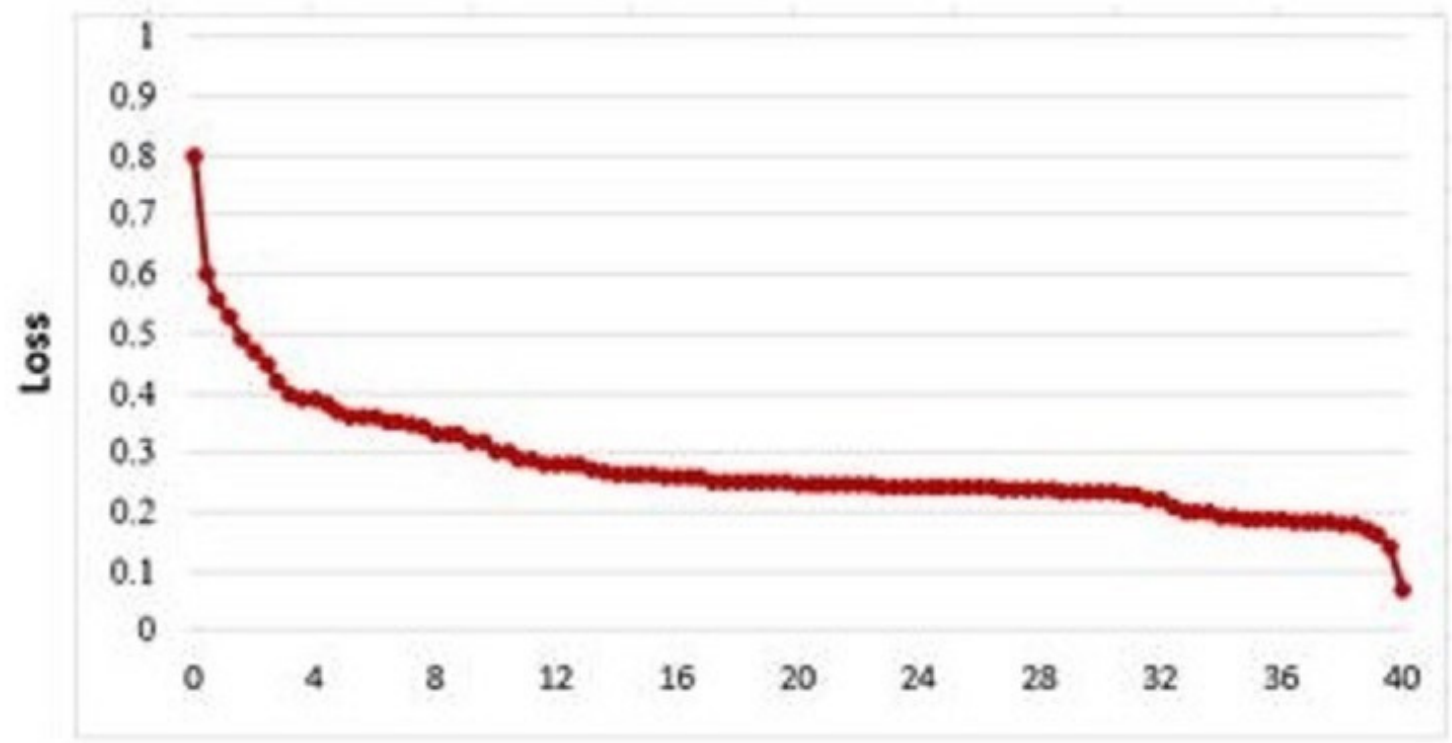

Epochs

(B) 


\section{Figure 4}

The saliency maps obtained from the proposed model and five other state-of-the-art models for a sample image from the TORONTO, MIT300, MIT1003, and DUT-OMRON datasets 
PeerJ Computer Science

\begin{tabular}{|c|c|c|c|c|c|c|}
\hline & \multicolumn{3}{|c|}{ TORONTO } & \multicolumn{3}{|c|}{ MIT300 } \\
\hline Model & Test image & $\begin{array}{c}\text { Ground } \\
\text { Truth }\end{array}$ & $\begin{array}{c}\text { Model } \\
\text { prediction }\end{array}$ & $\begin{array}{l}\text { Test } \\
\text { image }\end{array}$ & $\begin{array}{l}\text { Ground } \\
\text { Truth }\end{array}$ & $\begin{array}{c}\text { Model } \\
\text { prediction }\end{array}$ \\
\hline ITTI [7] & singes. & $\bullet$ & & & 4 & \\
\hline FES [11] & & $\boldsymbol{\bullet}$ & & & 4 & \\
\hline CovSal [12] & & $\bullet$ & & & 4 & \\
\hline GBVS [9] & & $\boldsymbol{\bullet}$ & & & 4 & ( \\
\hline $\begin{array}{c}\text { SDS-GM } \\
{[13]}\end{array}$ & wisk & $\bullet$ & & & , & \\
\hline Proposed & whe & - & & & , & ' \\
\hline & & MIT1003 & & & T-OMR & \\
\hline ITTI & 8 & & & & & \\
\hline FES & 9 & & & & & \\
\hline CovSal & 5 & & & & & \\
\hline GBVS & 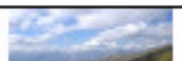 & & & & & \\
\hline SDS-GM & $Q^{20}$ & & & & & \\
\hline $\begin{array}{l}\text { Proposed } \\
\text { Model }\end{array}$ & Cos & & & - & 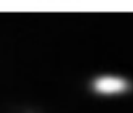 & $=$ \\
\hline
\end{tabular}




\section{Table $\mathbf{1}$ (on next page)}

Table 1. Configuration of the proposed model 
1

2

Table 1. Configuration of the proposed model.

3

4

\begin{tabular}{|c|c|c|}
\hline$======$ & Layer type & Filter size \\
\hline Encoder & Convolution & $3 \times 3,64$ \\
\hline & Residual Module & $(*), 64$ \\
\hline & Convolution & $3 \times 3,128$ \\
\hline & Max pooling & $2 \times 2$ \\
\hline & Convolution & $3 \times 3,256$ \\
\hline Decoder & Max pooling & $2 \times 2$ \\
\hline & Inception Module & $(*), 256$ \\
\hline & Transposed convolution & $3 \times 3,256$ \\
\hline & Convolution & $3 \times 3,256$ \\
\hline & Transposed convolution & $3 \times 3,64$ \\
\hline & Convolution & $3 \times 3,2$ \\
\hline & Pixel Classification Layer & - \\
\hline
\end{tabular}




\section{Table 2 (on next page)}

Table 1. Configuration of the proposed model 
1 Table 2. Configuration of inception and residual modules

2

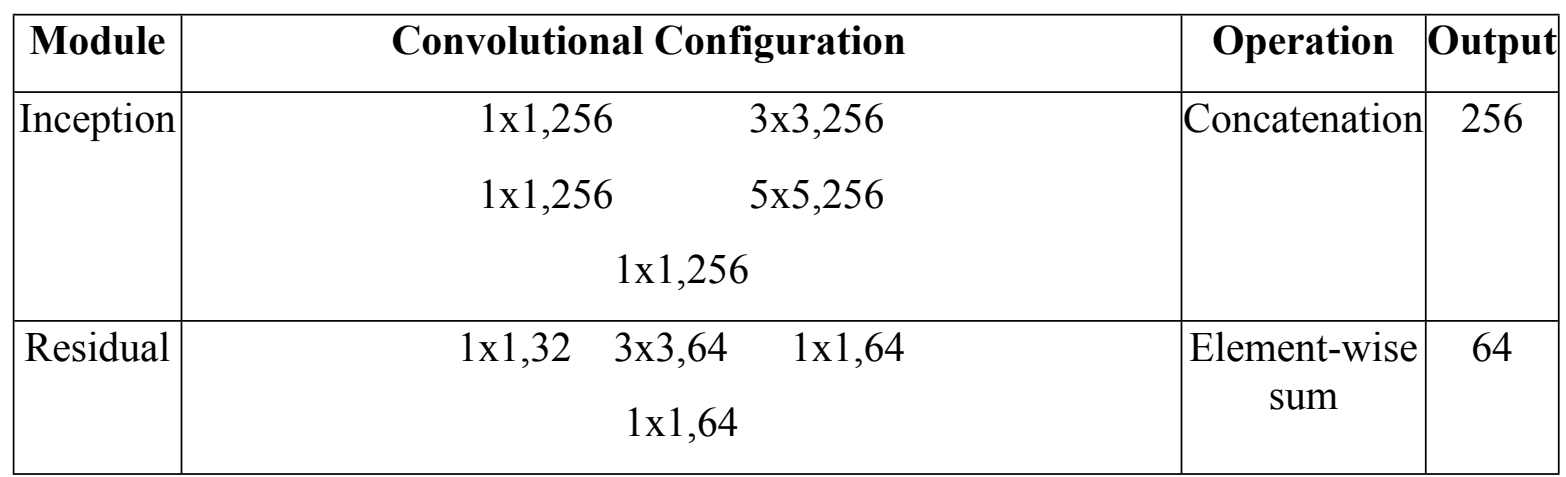

3 


\section{Table 3 (on next page)}

Table 3. Comparison of the quantitative scores of several models on TORONTO [20] dataset 
1 Table 3. Comparison of the quantitative scores of several models on TORONTO (Bruce \& 2 Tsotsos 2006) dataset.

\begin{tabular}{|c|c|c|c|c|}
\hline Model & NSS & SIM & AUC-Judd & AUC-Borji \\
\hline ITTI & 1.30 & 0.45 & 0.80 & 0.80 \\
\hline AIM & 0.84 & 0.36 & 0.76 & 0.75 \\
\hline Judd Model & 1.15 & 0.40 & 0.78 & 0.77 \\
\hline GBVS & 1.52 & 0.49 & 0.83 & 0.83 \\
\hline Mr-CNN & 1.41 & 0.47 & 0.80 & 0.79 \\
\hline CAS & 1.27 & 0.44 & 0.78 & 0.78 \\
\hline Proposed Model & 1.52 & 0.46 & 0.80 & 0.76 \\
\hline
\end{tabular}




\section{Table 4 (on next page)}

Table 4. Comparison of the quantitative scores of several models on MIT1003 [20] dataset 
1 Table 4. Comparison of the quantitative scores of several models on MIT300 (Judd et al. 2012) 2 dataset.

3

\begin{tabular}{|c|c|c|c|c|}
\hline Model & NSS & SIM & AUC-Judd & AUC-Borji \\
\hline ITTI (Itti et al. 1998) & 0.97 & 0.44 & 0.75 & 0.74 \\
\hline AIM (Bruce \& Tsotsos 2009) & 0.79 & 0.40 & 0.77 & 0.75 \\
\hline Judd Model (Judd et al. 2009) & 1.18 & 0.42 & 0.81 & 0.80 \\
\hline GBVS (Harel et al. 2007) & 1.24 & 0.48 & 0.81 & 0.80 \\
\hline Mr-CNN (Liu et al. 2016) & 1.13 & 0.45 & 0.77 & 0.76 \\
\hline CAS (Goferman et al. 2011) & 0.95 & 0.43 & 0.74 & 0.73 \\
\hline SalGAN (Pan et al. 2017) & 2.04 & 0.63 & 0.86 & 0.81 \\
\hline DeepGaze I (Kümmerer et al. 2014) & 1.22 & 0.39 & 0.84 & 0.83 \\
\hline DeepGaze II (Kummerer et al. 2017) & 1.29 & 0.46 & 0.87 & 0.86 \\
\hline ML-NET (Cornia et al. 2016) & 2.05 & 0.59 & 0.85 & 0.75 \\
\hline Proposed Model & 1.73 & 0.42 & 0.80 & 0.71 \\
\hline
\end{tabular}




\section{Table 5 (on next page)}

Table 5. Comparison of the quantitative scores of several models on MIT1003 [20] dataset 
1 Table 5. Comparison of the quantitative scores of several models on MIT1003(Judd et al. 2009) 2 dataset.

3

\begin{tabular}{|c|c|c|c|c|}
\hline Model & NSS & SIM & AUC-Judd & AUC-Borji \\
\hline ITTI & 1.10 & 0.32 & 0.77 & 0.76 \\
\hline AIM & 0.82 & 0.27 & 0.79 & 0.76 \\
\hline Judd Model & 1.18 & 0.42 & 0.81 & 0.80 \\
\hline GBVS & 1.38 & 0.36 & 0.83 & 0.81 \\
\hline Mr-CNN & 1.36 & 0.35 & 0.80 & 0.77 \\
\hline CAS & 1.07 & 0.32 & 0.76 & 0.74 \\
\hline SalGAN & 1.31 & 0.64 & 0.78 & 0.75 \\
\hline ML-NET & 1.64 & 0.35 & 0.82 & - \\
\hline Proposed Model & 1.35 & 0.44 & 0.88 & 0.78 \\
\hline
\end{tabular}




\section{Table 6(on next page)}

Table 6. Comparison of the quantitative scores of several models on DUT-OMRON [20] dataset 
1

2

3 Table 6. Comparison of the quantitative scores of several models on DUT-OMRON (Yang et al.

4

2013)

5

\begin{tabular}{|c|c|c|c|c|}
\hline Model & NSS & SIM & AUC-Judd & AUC-Borji \\
\hline ITTI & 3.09 & 0.53 & 0.83 & 0.83 \\
\hline AIM & 1.05 & 0.32 & 0.77 & 0.75 \\
\hline GBVS & 1.71 & 0.43 & 0.87 & 0.85 \\
\hline CAS & 1.47 & 0.37 & 0.80 & 0.79 \\
\hline Proposed Model & 1.84 & 0.45 & 0.88 & 0.76 \\
\hline
\end{tabular}




\section{Table 7 (on next page)}

Table 7. Different FCN models applied in this study 
1

2
Table 7: Runtime of the proposed model and ten visual saliency models

\begin{tabular}{|c|c|c|c|}
\hline Model & Training & Deep Learning & Run Time \\
\hline BMS & No & No & $0.3 \mathrm{~S}$ \\
\hline CAS & No & No & $16 \mathrm{~S}$ \\
\hline GBVS & No & No & $2 \mathrm{~S}$ \\
\hline ITTI & No & No & $4 \mathrm{~S}$ \\
\hline Mr-CNN & yes & Yes & $14 \mathrm{~S}(\mathrm{GPU})$ \\
\hline SalNet & yes & Yes & $0.1 \mathrm{~S}(\mathrm{GPU})$ \\
\hline eDn & yes & Yes & $8 \mathrm{~S}(\mathrm{GPU})$ \\
\hline AIM & yes & No & $2 \mathrm{~S}$ \\
\hline Judd Model & yes & No & $10 \mathrm{~S}$ \\
\hline DVA & yes & Yes & $0.1 \mathrm{~S}(\mathrm{GPU})$ \\
\hline Proposed Model & yes & Yes & $12 \mathrm{~S}$ \\
\hline
\end{tabular}




\section{Table 8 (on next page)}

Different FCN models applied in this study. 
Table 8. Different FCN models applied in this study.

2

\begin{tabular}{|c|c|c|c|c|c|}
\hline \multicolumn{2}{|r|}{ FCN Models } & \multicolumn{2}{|c|}{ Training } & \multicolumn{2}{|c|}{ Validation } \\
\hline Scenarios & Description & Accuracy & Loss & Accuracy & Loss \\
\hline S1 & 2 convolutional modules & $79.14 \%$ & 0.2650 & $78.88 \%$ & 0.2700 \\
\hline S2 & 3 convolutional modules & $85.05 \%$ & 0.2384 & $83.08 \%$ & 0.2571 \\
\hline S3 & 4 convolutional modules & $83.47 \%$ & 0.2548 & $82.94 \%$ & 0.2608 \\
\hline S4 & 5 convolutional modules \& & $80.04 \%$ & 0.2873 & $76.52 \%$ & 0.2775 \\
\hline S5 & 3 convolutional modules \& 1 inception modules & $89.69 \%$ & 0.2119 & $85.05 \%$ & 0.2231 \\
\hline S6 & 3 convolutional modules $\& 2$ inception modules & $90.84 \%$ & 0.1995 & $85.37 \%$ & 0.2454 \\
\hline S7 & 3 convolutional modules \& 3 inception modules & $93.63 \%$ & 0.1051 & $89.24 \%$ & 0.1666 \\
\hline S8 & 3 convolutional modules $\& 1$ residual modules & $87.55 \%$ & 0.2138 & $84.97 \%$ & 0.2317 \\
\hline S9 & 3 convolutional modules $\& 2$ residual modules & $83.23 \%$ & 0.2597 & $82.10 \%$ & 0.2684 \\
\hline S10 & 3 convolutional modules $\& 3$ residual modules & $81.66 \%$ & 0.2750 & $79.12 \%$ & 0.2921 \\
\hline S11 & $\begin{array}{l}3 \text { convolutional modules } \& 1 \text { inception module } \& 1 \\
\text { residual module }\end{array}$ & $89.46 \%$ & 0.1829 & $88.59 \%$ & 0.1889 \\
\hline S12 & $\begin{array}{l}3 \text { convolutional modules } \& 2 \text { inception module } \& 1 \\
\text { residual module }\end{array}$ & $92.73 \%$ & 0.1255 & $89.92 \%$ & 0.2111 \\
\hline S13 & $\begin{array}{l}3 \text { convolutional modules \& } 3 \text { inception module \& } 1 \\
\text { residual module }\end{array}$ & $97.05 \%$ & 0.07 & $90.64 \%$ & 0.1588 \\
\hline
\end{tabular}

